\title{
A hybrid predator-prey model with general functional responses under seasonal succession alternating between Gompertz and logistic growth
}

\author{
Lei Hang ${ }^{1}$, Long Zhang ${ }^{1 *}$ (D) Xiaowen Wang ${ }^{1}$, Hongli Li ${ }^{1}$ and Zhidong Teng ${ }^{1}$
}

"Correspondence:

longzhang_xj@sohu.com ${ }^{1}$ College of Mathematics and

System Sciences, Xinjiang

University, Urumqi, P.R. China

\section{Springer}

\begin{abstract}
In this paper, a hybrid predator-prey model with two general functional responses under seasonal succession is proposed. The model is composed of two subsystems: in the first one, the prey follows the Gompertz growth, and it turns to the logistic growth in the second subsystem since seasonal succession. The two processes are connected by impulsive perturbations. Some very general, weak criteria on the ultimate boundedness, permanence, existence, uniqueness and global attractivity of predator-free periodic solution are established. We find that the hybrid population model with seasonal succession has more survival possibilities of natural species than the usual population models. The theoretical results are illustrated by special examples and numerical simulations.
\end{abstract}

MSC: 92D25; 34D23

Keywords: Seasonal succession; Permanence; Periodic solution; Impulsive; General functional response

\section{Introduction}

The seasonal fluctuations of populations over time is due to the changes of environment in external (e.g., seasonal alternation, etc.) and internal (e.g., breeding, mating, predation, etc.). Theoretically, it has been shown that seasonality could influence both species growth and community structures $[1,2]$. Recently, many remarkable results with population models under seasonal succession have been obtained [3-10].

Generally, we choose hybrid models to describe global dynamics of species with seasonal succession. However, these hybrid models in non-equilibrium are more difficult to investigate mathematically than models in equilibrium for fewer analytical tools. Herb and Stefan [3] studied a macrophyte growth model based on process to observe the light limited growth of individual macrophyte and competition between two species. They found the significant effect of seasonal succession on biomass production. Levy et al. [4] studied a class of predator-prey-subsidy model in non-equilibrium and found the role of seasonality on predator-prey interactions. Klausmeier [5] obtained some important results on

(c) The Author(s) 2020. This article is licensed under a Creative Commons Attribution 4.0 International License, which permits use, sharing, adaptation, distribution and reproduction in any medium or format, as long as you give appropriate credit to the original author(s) and the source, provide a link to the Creative Commons licence, and indicate if changes were made. The images or other third party material in this article are included in the article's Creative Commons licence, unless indicated otherwise in a credit line to the material. If material is not included in the article's Creative Commons licence and your intended use is not permitted by statutory regulation or exceeds the permitted use, you will need to obtain permission directly from the copyright holder. To view a copy of this licence, visit http://creativecommons.org/licenses/by/4.0/. 
population model with seasonal variation. Jennifer et al. [6] found the significant impacts of two different seasons on the dynamics of the herbivore-plant defence system. Comparing these results mentioned above, we found that the analytic approaches on these ecological models with seasonal succession are limited.

Hsu and Zhao [7] studied the following single species model alternating between logistic growth and negative growth under seasonal succession:

$$
\begin{cases}\dot{x}=-\lambda x, & m \omega \leq t \leq m \omega+(1-\phi) \omega, \\ \dot{x}=r x\left[1-\frac{x}{k}\right], & m \omega+(1-\phi) \omega \leq t \leq(m+1) \omega,\end{cases}
$$

where $\lambda, r, k$ are positive constants, $r$ and $k$ denote the intrinsic growth rate of species $x$ and the environment capacity, respectively, $m \in N, \phi \in(0,1]$. The system periodically alternates between two continuous seasons (season 1 and season 2), i.e., the temporal interval $[m \omega, m \omega+(1-\phi) \omega]$ and $[m \omega+(1-\phi) \omega,(m+1) \omega]$ represent season 1 and season 2 , respectively. The system is in season 1 during $[m \omega, m \omega+(1-\phi) \omega]$ and turns to season 2 without loss at time $m \omega+(1-\phi) \omega$. If the environment changes again, it will turn back to season 1 at time $(m+1) \omega$. A number of results on the global dynamics of system (1) were obtained including the convergence of forward orbits, stability of semi-trivial and positive fixed points, and nonexistence and uniqueness of positive fixed point for the discrete-time dynamical system.

It is well known that the dynamics of population growth can be usually depicted by two main kinds of equations. One is the logistic equation, various significant findings about population models with logistic growth have been found (see, e.g., [11-13]). The other is the Gompertz equation, which has been proven to be a simple method to produce asymmetrical types of S-shaped curves [14]. Actually, the Gompertz and logistic curves are both "S-shaped" and can be used to describe population dynamics processes. Compared with them, we can find that they are vastly different in the site of inflection point and the Gompertz curve reaches the maximum rate of growth earlier than the latter.

In general, ecosystems are affected by temporal and spatial variation, human activity. Therefore, the system may experience a sudden interference tautologically. Several kinds of real processes can be described by impulsive differential equation, such as prey impulsive diffusion [15, 16], birth pulses [17] and impulsively biological control [18].

$\mathrm{Li}$ and Zhang [10] studied the following single species model under seasonal succession with impulsive perturbations alternating between Gompertz and logistic equations:

$$
\begin{cases}\dot{x}=r_{1} x \ln \frac{k_{1}}{x}, & m \omega<t \leq m \omega+(1-\phi) \omega, \\ x\left(t^{+}\right)=\alpha_{1} x(t), & t=m \omega+(1-\phi) \omega, \\ \dot{x}=r_{2} x\left[1-\frac{x}{k_{2}}\right], & m \omega+(1-\phi) \omega<t \leq(m+1) \omega, \\ x\left(t^{+}\right)=\alpha_{2} x(t), & t=(m+1) \omega,\end{cases}
$$

where $r_{i}$ and $k_{i}$ represent the intrinsic growth rate and environment capacity of the population in season $i(i=1,2)$, respectively. $x\left(t^{+}\right)=\lim _{t \rightarrow t^{+}} x(t)$ represents the population density of species $x$ at the time of impulsive point $t=m \omega+(1-\phi) \omega($ or $t=(m+1) \omega)$ $(m=0,1,2, \ldots)$. Biologically, $\alpha_{i}>0(i=1,2)$, and particularly, while $\alpha_{i}>1\left(\alpha_{i}<1\right)$, the population density increases (or decreases) proportionally. Criteria on the permanence, exis- 
tence, uniqueness and global stability of positive $\omega$-periodic solution of system (2) were given.

Hsu and Zhao [7] further studied the following two-species Lotka-Volterra competition model under seasonal succession:

$$
\begin{cases}\dot{x}_{i}(t)=-\lambda_{i} x_{i}(t), & m \omega \leq t \leq m \omega+(1-\phi) \omega, i=1,2, \\ \dot{x}_{1}(t)=r_{1} x_{1}(t)\left[1-\frac{x_{1}}{K_{1}}\right]-\alpha x_{1} x_{2}, & m \omega+(1-\phi) \omega \leq t \leq(m+1) \omega, \\ \dot{x}_{2}(t)=r_{2} x_{2}(t)\left[1-\frac{x_{2}}{K_{2}}\right]-\beta x_{1} x_{2}, & m \omega+(1-\phi) \omega \leq t \leq(m+1) \omega .\end{cases}
$$

Criteria on the whole global dynamics including the global extinction of both species, the competitive exclusion, the competitive coexistence of two species, and the saddle-point structure were obtained.

Based on above consideration, we propose a hybrid predator-prey model with general functional responses under seasonal succession alternating between the Gompertz and logistic growths of prey connected by impulsive perturbations as follows:

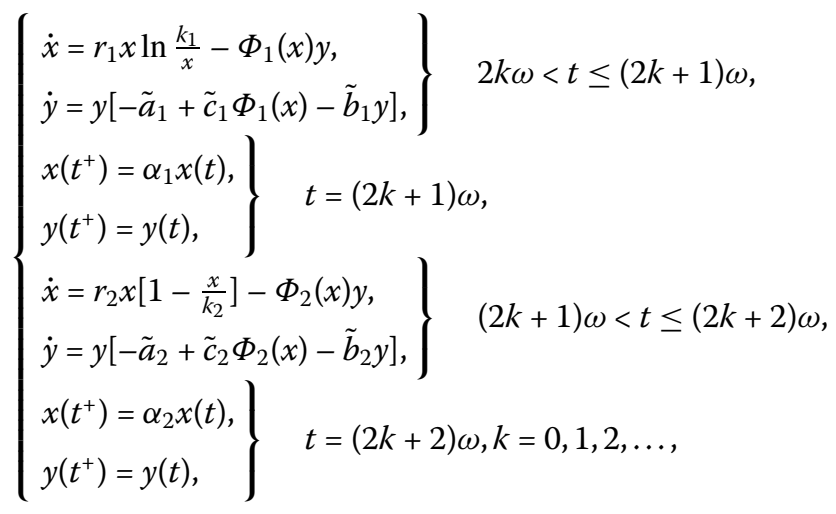

where $\tilde{a}_{i}$ and $\tilde{b}_{i}$ denote the parameters of death rate and density dependence of the predator species $y$. Here, $\Phi_{i}(x)(i=1,2)$ are the general functional responses of predation. The system consists of two alternating seasons (season 1 and 2) switching periodically, i.e., the population $x$ and $y$ live in season 1 during $(2 k \omega,(2 k+1) \omega], x\left(t^{+}\right)=\lim _{t \rightarrow t^{+}} x(t)$ and $y\left(t^{+}\right)=\lim _{t \rightarrow t^{+}} y(t)$ represent the prey and predator population at the time of impulsive point $t=(2 k+1) \omega$, respectively. After this point, population $x$ and $y$ turn to live in season 2 over $((2 k+1) \omega,(2 k+2) \omega]$, and $x\left(t^{+}\right)=\lim _{t \rightarrow t^{+}} x(t)$ and $y\left(t^{+}\right)=\lim _{t \rightarrow t^{+}} y(t)$ denote the prey and predator population at the time of impulsive point $t=(2 k+2) \omega$, respectively, then the system completes one cycle. With the changes of environment again, it turns back to season 1 at time $(2 k+2) \omega$, then it repeats the above processes. In this paper, we assume that $r_{1}, k_{1}, r_{2}, k_{2}, \tilde{a}_{i}, \tilde{b}_{i}$ and $\alpha_{i}(i=1,2)$ are all positive constants.

In this paper, our main goal is to study the global dynamics of system (4). Criteria on the permanence, existence, uniqueness and global attractivity of nonnegative periodic solution of system (4) are established. From our results, we find that the hybrid population model with seasonal succession has very significant impact on the dynamical properties of natural species, it permits more survival possibilities of life in a real ecosystem than the usual population models.

The outline of our work is as follows. In Sect. 2, we give some useful hypotheses, lemmas with respect to the stability of $\omega$-periodic solution. In Sect. 3, we investigate the ultimate 
boundedness, permanence, existence, uniqueness and global attractivity of nonnegative periodic solutions for system (4). In Sect. 4, the main results are illustrated and discussed by some special examples and numerical simulations.

\section{Preliminaries}

The solution of model (4) denoted by $(x(t), y(t))$ is piecewise-continuous on $(2 k \omega, 2(k+$ 1) $\omega](k=0,1,2, \ldots)$, and $x\left(t^{+}\right)=\lim _{t \rightarrow t^{+}} x(t)$ exists, where $t=(2 k+1) \omega($ or $(2 k+2) \omega)$. Clearly, the existence and uniqueness of solution of model (4) can be guaranteed by the smoothness properties of the right-hand side of model (4) ([19-22]). The proof of the positivity of any solution $(x(t), y(t))$ of model (4) with any initial value $(x(0), y(0)) \in R_{+}^{2}=\{(x, y) \mid$ $x>0, y>0\}$ is so easy that we omit it.

First, we introduce the following hypothesis for model (4):

$\left(H_{1}\right): \Phi_{i}(x)(i=1,2)$ is strictly increasing with $x \in R_{+}=[0,+\infty)$ and continuous differentiable with $x, \Phi_{i}(0)=0$.

Remark 2.1 Obviously, Holling type I, II, III and IV functional responses are special cases of the general functional response $\Phi_{i}(x)(i=1,2)$.

Next, for convenience of the discussion, we set $a_{2}=r_{2}, b_{2}=\frac{r_{2}}{k_{2}}$, we can rewrite model (2) into

$$
\begin{cases}\dot{x}=r_{1} x \ln \frac{k_{1}}{x}, & 2 k \omega<t \leq(2 k+1) \omega \\ x\left(t^{+}\right)=\alpha_{1} x(t), & t=(2 k+1) \omega \\ \dot{x}=x\left(a_{2}-b_{2} x\right), & (2 k+1) \omega<t \leq(2 k+2) \omega \\ x\left(t^{+}\right)=\alpha_{2} x(t), & t=(2 k+2) \omega, k=0,1,2, \ldots\end{cases}
$$

we can further rewrite model (5) as follows:

$$
\begin{cases}\dot{x}=\frac{(-1)^{k}+1}{2} r_{1} x \ln \frac{k_{1}}{x}+\frac{(-1)^{k+1}+1}{2} x\left(a_{2}-b_{2} x\right), & t \in(k \omega,(k+1) \omega] \\ x\left(t^{+}\right)=\frac{(-1)^{k}+1}{2} \alpha_{1} x(t)+\frac{(-1)^{k+1}+1}{2} \alpha_{2} x(t), & t=(k+1) \omega, k \in N .\end{cases}
$$

Furthermore, we introduce the following assumption for model (5) (or (6)):

$\left(H_{2}\right)$ :

$$
\frac{\left(\frac{a_{2}}{b_{2}}\right)^{1-a}}{k_{1}^{1-a} b^{a}(b-1)^{1-a}}<\alpha_{1} \alpha_{2}^{a} \leq 1
$$

where $0<a=e^{-r_{1} \omega}<1, b=e^{a_{2} \omega}>1$.

For system (5) (or (6)), we have the following result.

Lemma 2.1 (See [7]) If $\left(H_{2}\right)$ hold, then system (5) (or (6)) has a unique positive $2 \omega$-periodic solution $u_{0}^{*}(t)$, which is globally asymptotically stable.

Finally, we consider the following scalar impulsive differential equation:

$$
\begin{cases}\frac{d x(t)}{d t}=f(t, x(t)), & t \neq t_{k} \\ x\left(t_{k}^{+}\right)=I\left(x\left(t_{k}\right)\right), & t=t_{k}\end{cases}
$$


where $t \in R_{+}, 0 \leq t_{1}<t_{2}<\cdots<t_{k}<t_{k+1}<\cdots$ is impulsive time sequence, $x \in R, f(x, x)$ : $R_{+} \times R \rightarrow R$ is continuous and $I(x): R \rightarrow R$ is a non-decreasing function. We have the following comparison theorem for Eq. (7).

Lemma 2.2 (See [19]) Let $x(t)$ be a solution of system (7) defined on $\left[t_{0}, T\right]$ and the function $u(t)$ be defined on $\left[t_{0}, T\right]$ and satisfy

$$
\begin{cases}\frac{d u(t)}{d t} \leq(\geq) f(t, u(t)), & t \neq t_{k} \\ u\left(t_{k}^{+}\right) \leq(\geq) I\left(u\left(t_{k}\right)\right), & t=t_{k} .\end{cases}
$$

If $u\left(t_{0}\right) \leq(\geq) x\left(t_{0}\right)$, then $u(t) \leq(\geq) x(t)$ for all $t \in\left[t_{0}, T\right]$.

\section{Main results}

First, as regards the ultimately upper boundedness of model (4), we have the following.

Theorem 3.1 If $\left(H_{1}\right)-\left(H_{2}\right)$ hold, then there exists a constant $M>0$, such that, for any positive solution $(x(t), y(t))$ of model (4), we have

$$
\lim _{t \rightarrow \infty} \sup x(t) \leq M, \quad \lim _{t \rightarrow \infty} \sup y(t) \leq M
$$

Proof Let $(x(t), y(t))$ be any positive solution of model (4), we directly obtain for any $t \geq 0$

$$
\begin{cases}\dot{x} \leq \frac{(-1)^{k}+1}{2} r_{1} x \ln \frac{k_{1}}{x}+\frac{(-1)^{k+1}+1}{2} x\left(a_{2}-b_{2} x\right), & t \in(k \omega,(k+1) \omega], \\ x\left(t^{+}\right)=\frac{(-1)^{k}+1}{2} \alpha_{1} x(t)+\frac{(-1)^{k+1}+1}{2} \alpha_{2} x(t), & t=k \omega, k \in N .\end{cases}
$$

Consider the following auxiliary equation:

$$
\begin{cases}\dot{u}=\frac{(-1)^{k}+1}{2} r_{1} u \ln \frac{k_{1}}{u}+\frac{(-1)^{k+1}+1}{2} u\left(a_{2}-b_{2} u\right), & t \in(k \omega,(k+1) \omega], \\ u\left(t^{+}\right)=\frac{(-1)^{k}+1}{2} \alpha_{1} u(t)+\frac{(-1)^{k+1}+1}{2} \alpha_{2} u(t), & t=k \omega, k \in N\end{cases}
$$

from Lemma 2.2, we have $x(t) \leq u(t)$ for all $t \geq 0$, where $u(t)$ is the solution of system (10) with initial value $u(0)=x(0)$. By Lemma 2.1 , system (10) has a unique globally asymptotically stable positive $2 \omega$-periodic solution $u_{0}^{*}(t)$. Therefore, for any constant $\eta_{1}>0$, there exists a constant $T_{1}>0$ such that $u(t)<u^{*}(t)+\eta_{1}, t \geq T_{1}$. Hence,

$$
x(t) \leq u(t)<u^{*}(t)+\eta_{1} \leq \max _{t \in[0,2 \omega]} u_{0}^{*}(t)+\eta_{1} \triangleq M_{1},
$$

for all $t \geq T_{1}$. Therefore, from system (4) and $\left(H_{1}\right)$, we can obtain

$$
\left\{\begin{aligned}
\dot{y} \leq & \frac{(-1)^{k}+1}{2} y\left[\tilde{c}_{1} \Phi_{1}\left(\max _{t \in[0,2 \omega]} u_{0}^{*}(t)+\eta_{1}\right)-\tilde{b}_{1} y\right] & \\
\quad+\frac{(-1)^{k+1}+1}{2} y\left[\tilde{c}_{2} \Phi_{2}\left(\max _{t \in[0,2 \omega]} u_{0}^{*}(t)+\eta_{1}\right)-\tilde{b}_{2} y\right], & & t \in(k \omega,(k+1) \omega] \\
y\left(t^{+}\right)=\frac{(-1)^{k}+1}{2} y(t)+\frac{(-1)^{k+1}+1}{2} y(t), & & t=k \omega, k \in N .
\end{aligned}\right.
$$


From Lemma 2.2, we have $y(t) \leq v(t)$, where $v(t)$ is the solution of the following auxiliary system with initial value $v(0)=y(0)$ :

$$
\begin{cases}\dot{v}=v(A-B v), & t \in(k \omega,(k+1) \omega] \\ v\left(t^{+}\right)=v(t), & t=k \omega, k \in N\end{cases}
$$

where

$$
\begin{aligned}
& A=\frac{(-1)^{k}+1}{2}\left[\tilde{c}_{1} \Phi_{1}\left(M_{1}\right)\right]+\frac{(-1)^{k+1}+1}{2}\left[\tilde{c}_{2} \Phi_{2}\left(M_{1}\right)\right]>0, \\
& B=\frac{(-1)^{k}+1}{2} \tilde{b}_{1}+\frac{(-1)^{k+1}+1}{2} \tilde{b}_{2}>0 .
\end{aligned}
$$

Distinctly, system (13) has a unique globally asymptotically stable positive equilibrium $v^{*}=\frac{A}{B}$. Therefore, for any constant $\eta_{2}>0$, there exists a constant $T_{2}>T_{1}$ such that $v(t) \leq$ $\frac{A}{B}+\eta_{2}, t \geq T_{2}$. Hence,

$$
y(t) \leq v(t) \leq \frac{A}{B}+\eta_{2} \triangleq M_{2}
$$

for all $t \geq T_{2}$. Let $M=\max \left\{M_{1}, M_{2}\right\}$, therefore, we have

$$
\lim _{t \rightarrow \infty} \sup x(t) \leq M, \quad \lim _{t \rightarrow \infty} \sup y(t) \leq M
$$

for all $t \geq T_{2}$. This completes the proof of Theorem 3.1.

From $\left(H_{1}\right)$ and the mean-value theorem, we see that there exist a $\xi_{1} \in(0, x(t))$ and a $\xi_{2} \in(0, x(t))$ such that

$$
\Phi_{1}(x(t))=\Phi_{1}(x(t))-\Phi_{1}(0)=\dot{\Phi}_{1}\left(\xi_{1}\right) x(t)
$$

and

$$
\Phi_{2}(x(t))=\Phi_{2}(x(t))-\Phi_{2}(0)=\dot{\Phi}_{2}\left(\xi_{2}\right) x(t) .
$$

Consider the following system:

$$
\begin{cases}\dot{u}=u\left(r_{1} \ln \frac{k_{1}}{u}-\xi\right), & t \in(2 k \omega,(2 k+1) \omega], \\ u\left(t^{+}\right)=\alpha_{1} u(t), & t=(2 k+1) \omega \\ \dot{u}=u\left(a_{2}-b_{2} u-\xi\right), & t \in((2 k+1) \omega,(2 k+2) \omega], \\ u\left(t^{+}\right)=\alpha_{2} u(t), & t=(2 k+2) \omega,\end{cases}
$$

where $\xi=\max _{0<x \leq M}\left\{\dot{\Phi}_{1}(x) M, \dot{\Phi}_{2}(x) M\right\}, M$ is defined in Theorem 3.1. 
Let $z(t)=\exp ^{\frac{\xi}{r_{1}}} u(t)$, we can rewrite model (17) as

$$
\begin{cases}\dot{z}=r_{1} z \ln \frac{k_{1}}{z}, & t \in(2 k \omega,(2 k+1) \omega] \\ z\left(t^{+}\right)=\alpha_{1} z(t), & t=(2 k+1) \omega \\ \dot{z}=z(\bar{a}-\bar{b} z), & t \in((2 k+1) \omega,(2 k+2) \omega] \\ z\left(t^{+}\right)=\alpha_{2} z(t), & t=(2 k+2) \omega .\end{cases}
$$

Here $\bar{a}=a_{2}-\xi, \bar{b}=b_{2} e^{-\frac{\xi}{r_{1}}}$. For model (18), we further introduce the following assumption: $\left(H_{3}\right)$ :

$$
\frac{\left(\frac{\bar{a}}{\bar{b}}\right)^{1-a}}{k_{1}^{1-a} \tilde{b}^{a}(\tilde{b}-1)^{1-a}}<\alpha_{1} \alpha_{2}^{a} \leq 1
$$

where $0<a=e^{-r_{1} \omega}<1, \tilde{b}=e^{\bar{a} \omega}>1$.

As a consequence of Lemma 2.1, we have the following result.

Corollary 3.2 If $\left(\mathrm{H}_{3}\right)$ hold, then system (17) has a unique positive $2 \omega$-periodic solution $u_{\xi}^{*}(t)$, which is globally asymptotically stable, and

$$
\lim _{\xi \rightarrow 0} u_{\xi}^{*}(t)=u_{0}^{*}(t)
$$

where $u_{0}^{*}(t)$ is the unique periodic solution of model (5).

Proof From Lemma 2.1, we have system (17) has a unique positive $2 \omega$-periodic solution $u_{\xi}^{*}(t)$, which is globally asymptotically stable. Because the right-hand side of Eq. (18) satisfies the local Lipschitz condition with respect to $z(t)$, according to the continuity with respect to the parameters of solution of impulsive differential equations, we can find that any solution $z\left(t, t_{0}, z_{0}, \xi\right)$ of Eq. (18) with initial condition $z_{0}=x_{0}$, is continuous with $\left(t_{0}, z_{0}, \xi\right)$ (Theorems 2.9 and 2.10 given in Chap. 1 in [20]). Furthermore, we find that the unique positive $2 \omega$-periodic solution $u_{\xi}^{*}(t)$ of model (17) is continuous with respect to the parameter $\xi$. Therefore, we finally have

$$
\lim _{\xi \rightarrow 0} u_{\xi}^{*}(t)=u_{0}^{*}(t)
$$

This completes the proof of Corollary 3.2.

Next, we have the following result with respect to the permanence of model (4).

Theorem 3.3 If $\left(H_{1}\right)-\left(H_{3}\right)$ hold, and

$$
\int_{0}^{\omega}\left[-\tilde{a}_{1}+\tilde{c}_{1} \Phi_{1}\left(u_{0}^{*}(t)\right)\right] d t+\int_{\omega}^{2 \omega}\left[-\tilde{a}_{2}+\tilde{c}_{2} \Phi_{2}\left(u_{0}^{*}(t)\right)\right] d t>0
$$

then model (4) is permanent, i.e., there exist constants $M>m>0$ such that, for any positive solution $(x(t), y(t))$ of model (4), we have

$$
m \leq \lim _{t \rightarrow \infty} \inf x(t) \leq \lim _{t \rightarrow \infty} \sup x(t) \leq M
$$


and

$$
m \leq \lim _{t \rightarrow \infty} \inf y(t) \leq \lim _{t \rightarrow \infty} \sup y(t) \leq M
$$

Proof From Theorem 3.1, we only need to prove that there exists a constant $m>0$ such that, for any positive solution $(x(t), y(t))$ of system (4), there is a positive constant $\bar{T}>T_{2}$, when $t>\bar{T}$, we have

$$
x(t)>m, \quad y(t)>m .
$$

Let $(x(t), y(t))$ be any positive solution of system (4), from Theorem 3.1, there is a constant $T_{2}>T_{1}$ such that

$$
x(t) \leq M, \quad y(t) \leq M,
$$

for all $t \geq T_{2}$. For all $t \geq T_{2}$, from the mean-value theorem, we see that there exist a $\xi_{1} \in$ $(0, x(t))$ and $\mathrm{a} \xi_{2} \in(0, x(t))$ such that

$$
\Phi_{1}(x(t))=\Phi_{1}(x(t))-\Phi_{1}(0)=\dot{\Phi}_{1}\left(\xi_{1}\right) x(t)
$$

and

$$
\Phi_{2}(x(t))=\Phi_{2}(x(t))-\Phi_{2}(0)=\dot{\Phi}_{2}\left(\xi_{2}\right) x(t) .
$$

From system (4), for any $t \geq T_{2}$, we have

$$
\begin{cases}\dot{x} \geq x\left(r_{1} \ln \frac{k_{1}}{x}-\xi\right), & t \in(2 k \omega,(2 k+1) \omega], \\ x\left(t^{+}\right)=\alpha_{1} x(t), & t=(2 k+1) \omega, \\ \dot{x} \geq x\left(a_{2}-b_{2} x-\xi\right), & t \in((2 k+1) \omega,(2 k+2) \omega], \\ x\left(t^{+}\right)=\alpha_{2} x(t), & t=(2 k+2) \omega .\end{cases}
$$

Here $\xi=\max _{0<x \leq M}\left\{\dot{\Phi}_{1}(x) M, \dot{\Phi}_{2}(x) M\right\}$.

From Lemma 2.2, we have $x(t) \geq u(t)$, where $u(t)$ is the solution of the following system (22) with initial condition $u\left(T_{2}^{+}\right)=x\left(T_{2}^{+}\right)$.

$$
\begin{cases}\dot{u}=u\left(r_{1} \ln \frac{k_{1}}{u}-\xi\right), & t \in(2 k \omega,(2 k+1) \omega], \\ u\left(t^{+}\right)=\alpha_{1} u(t), & t=(2 k+1) \omega, \\ \dot{u}=u\left(a_{2}-b_{2} u-\xi\right), & t \in((2 k+1) \omega,(2 k+2) \omega], \\ u\left(t^{+}\right)=\alpha_{2} u(t), & t=(2 k+2) \omega .\end{cases}
$$

Let $z(t)=\exp ^{\frac{\xi}{r_{1}}} u(t)$, we can obtain

$$
\begin{cases}\dot{z}=r_{1} z \ln \frac{k_{1}}{z}, & t \in(2 k \omega,(2 k+1) \omega], \\ z\left(t^{+}\right)=\alpha_{1} z(t), & t=(2 k+1) \omega, \\ \dot{z}=z(\bar{a}-\bar{b} z), & t \in((2 k+1) \omega,(2 k+2) \omega], \\ z\left(t^{+}\right)=\alpha_{2} z(t), & t=(2 k+2) \omega .\end{cases}
$$


Here $\bar{a}=a_{2}-\xi, \bar{b}=b_{2} e^{-\frac{\xi}{r_{1}}}$. By Corollary 3.2, system (26) has a unique globally asymptotically stable positive $2 \omega$-periodic solution $z^{*}(t)$, i.e., system (25) has a unique globally asymptotically stable positive $2 \omega$-periodic solution $u_{\xi}^{*}(t)$.

For $\eta=\frac{1}{2} \min _{t \in[0,2 \omega]} u_{\xi}^{*}(t)$, there exists a $\bar{T}_{2}>T_{2}$ such that $u(t) \geq u_{\xi}^{*}(t)-\eta$, for any $t \geq \bar{T}_{2}$. Hence,

$$
x(t) \geq u(t) \geq u_{\xi}^{*}(t)-\eta \geq \frac{1}{2} \min _{t \in[0,2 \omega]} u_{\xi}^{*}(t) \triangleq \eta=m_{1},
$$

for any $t \geq \bar{T}_{2}$. This shows that $x(t)$ is permanent.

Next we prove that $y(t)$ is permanent. From condition (19), we can choose a small enough constant $\epsilon_{0}>0$ such that

$$
\begin{aligned}
\delta:= & \int_{0}^{\omega}\left[-\tilde{a}_{1}+\tilde{c}_{1} \Phi_{1}\left(u_{0}^{*}(v)-\epsilon_{0}\right)-\tilde{b}_{1} \epsilon_{0}\right] d v \\
& +\int_{\omega}^{2 \omega}\left[-\tilde{a}_{2}+\tilde{c}_{2} \Phi_{2}\left(u_{0}^{*}(v)-\epsilon_{0}\right)-\tilde{b}_{2} \epsilon_{0}\right] d v
\end{aligned}
$$

$>0$.

From Corollary 3.2, we also have

$$
\lim _{\xi \rightarrow 0} u_{\xi}^{*}(t)=u_{0}^{*}(t)
$$

Therefore, for above constant $\epsilon_{0}$, there is a constant $\xi_{0}>0$ such that

$$
u_{\xi_{0}}^{*}(t) \geq u_{0}^{*}(t)-\frac{\epsilon_{0}}{2}
$$

for all $t \geq 0$, where $u_{\xi_{0}}^{*}(t)$ and $u_{0}^{*}(t)$ are the unique positive periodic solutions of systems (26) and (5) with $\xi=\xi_{0}$, respectively. Because $u_{\xi_{0}}^{*}(t)$ is the unique globally asymptotically stable positive $2 \omega$-periodic solution of system (22) with $\xi=\xi_{0}$, for $\epsilon_{0}, M$ and any initial value $\left(t_{0}, u_{0}\right)$ with $t_{0} \geq 0$ and $0<u_{0}<M$, there is a constant $\tilde{T}_{1}=\tilde{T}_{1}\left(\epsilon_{0}, M, u_{0}\right)>0$ such that

$$
\left|u(t)-u_{\xi_{0}}^{*}(t)\right| \leq \frac{\epsilon_{0}}{2}
$$

for all $t \geq t_{0}+\tilde{T}_{1}$, where $u(t)$ is the positive solution of system (22) with $\xi=\xi_{0}$ and initial condition $u\left(t_{0}^{+}\right)=u_{0}$. Hence, we furthermore have

$$
u(t) \geq u_{\xi_{0}}^{*}(t)-\frac{\epsilon_{0}}{2}
$$

for all $t \geq t_{0}+\tilde{T}_{1}$.

Let $\epsilon_{1}=\min \left\{\frac{\xi_{0}}{\tilde{M}}, \epsilon_{0}\right\}$, where $\tilde{M}=\max \left\{\dot{\Phi}_{1}(M), \dot{\Phi}_{2}(M)\right\}$, we now consider $y(t)$. There exist three cases as follows. 
Case 1 , there is a constant $T^{*} \geq \tilde{T}_{2}$ such that $y(t) \leq \epsilon_{1}$ for all $t \geq T^{*}$.

Case 2, there is a constant $T^{*} \geq \tilde{T}_{2}$ such that $y(t) \geq \epsilon_{1}$ for all $t \geq T^{*}$.

Case 3, there is an interval sequence $\left\{\left[s_{n}, t_{n}\right]\right\}$ with $T_{2} \leq s_{1}<t_{1}<s_{2}<t_{2}<\cdots<s_{n}<t_{n}<$ $\cdots$ and $\lim _{n \rightarrow \infty} s_{n}=\infty$ such that $y(t) \leq \epsilon_{1}$ for all $t \in \bigcup_{n=1}^{\infty}\left[s_{n}, t_{n}\right], y\left(s_{n}\right)=y\left(t_{n}\right)=\epsilon_{1}$ and $y(t) \geq \epsilon_{1}$ for all $t \notin \bigcup_{n=1}^{\infty}\left(s_{n}, t_{n}\right)$.

We firstly consider case 1 , for any $t \geq \tilde{T}_{2}$, we have

$$
\begin{cases}\dot{x} \geq x\left(r_{1} \ln \frac{k_{1}}{x}-\xi_{0}\right), & t \in(2 k \omega,(2 k+1) \omega], \\ x\left(t^{+}\right)=\alpha_{1} x(t), & t=(2 k+1) \omega, \\ \dot{x} \geq x\left(a_{2}-b_{2} x-\xi_{0}\right), & t \in((2 k+1) \omega,(2 k+2) \omega], \\ x\left(t^{+}\right)=\alpha_{2} x(t), & t=(2 k+2) \omega .\end{cases}
$$

From (27), (30) and (32), we can obtain, when $t \geq \tilde{T}_{2}+\tilde{T}_{1}$,

$$
x(t) \geq u(t) \geq u_{\xi_{0}}^{*}(t)-\frac{\epsilon_{0}}{2} \geq u_{0}^{*}(t)-\epsilon_{0} .
$$

When $t \geq \tilde{T}_{2}+\tilde{T}_{1}$, we furthermore have

$$
\begin{array}{r}
\dot{y}(t) \geq y(t)\left[\frac{(-1)^{k}+1}{2}\left(-\tilde{a}_{1}+\tilde{c}_{1} \Phi_{1}\left(u_{0}^{*}(t)-\epsilon_{0}\right)-\tilde{b}_{1} \epsilon_{0}\right)\right. \\
\left.+\frac{(-1)^{k+1}+1}{2}\left(-\tilde{a}_{2}+\tilde{c}_{2} \Phi_{2}\left(u_{0}^{*}(t)-\varepsilon_{0}\right)-\tilde{b}_{2} \epsilon_{0}\right)\right] .
\end{array}
$$

For any integer $k \geq 0$, we take $t \in\left[T_{3}+2 k \omega, T_{3}+(2 k+1) \omega\right)$, where $T_{3}=\tilde{T}_{2}+\tilde{T}_{1}$, integrating (35) from $T_{3}$ to $t$, by (28), we can obtain

$$
\begin{aligned}
y(t) \geq & y\left(T_{3}\right) \exp \left\{\int_{T_{3}}^{T_{3}+\omega}\left[-\tilde{a}_{1}+\tilde{c}_{1} \Phi_{1}\left(u_{0}^{*}(v)-\epsilon_{0}\right)-\tilde{b}_{1} \epsilon_{0}\right] d v\right. \\
& +\int_{T_{3}+\omega}^{T_{3}+2 \omega}\left[-\tilde{a}_{2}+\tilde{c}_{2} \Phi_{2}\left(u_{0}^{*}(v)-\epsilon_{0}\right)-\tilde{b}_{2} \epsilon_{0}\right] d v \\
& +\cdots+ \\
& +\int_{T_{3}+(2 k-2) \omega}^{T_{3}+(2 k-1) \omega}\left[-\tilde{a}_{1}+\tilde{c}_{1} \Phi_{1}\left(u_{0}^{*}(v)-\epsilon_{0}\right)-\tilde{b}_{1} \epsilon_{0}\right] d v \\
& +\int_{T_{3}+(2 k-1) \omega}^{T_{3}+2 k \omega}\left[-\tilde{a}_{2}+\tilde{c}_{2} \Phi_{2}\left(u_{0}^{*}(v)-\epsilon_{0}\right)-\tilde{b}_{2} \epsilon_{0}\right] d v \\
& \left.+\int_{T_{3}+2 k \omega}^{t}\left[-\tilde{a}_{1}+\tilde{c}_{1} \Phi_{1}\left(u_{0}^{*}(v)-\epsilon_{0}\right)-\tilde{b}_{1} \epsilon_{0}\right] d v\right\} \\
\geq & y\left(T_{3}\right) \exp (k \delta-\beta \omega) .
\end{aligned}
$$

Here, $\beta=\max _{t \in\left[T_{3}+2 k \omega, T_{3}+(2 k+1) \omega\right]}\left\{\tilde{a}_{1}+\tilde{c}_{1} \Phi_{1}\left(u_{0}^{*}(t)-\epsilon_{0}\right)+\tilde{b}_{1} \epsilon_{0}\right\}$. From this, we further obtain $y(t) \rightarrow+\infty$ as $k \rightarrow \infty$, which leads to a contradiction. Therefore, case 1 cannot hold. 
Now we consider case 3. For any $t \geq T_{2}$, when $t \in \bigcup_{n=1}^{\infty}\left[s_{n}, t_{n}\right]$, then $t \in\left[s_{n}, t_{n}\right]$ for some $n$. If $t_{n}-s_{n} \leq \tilde{T}_{1}$, then because $\epsilon_{1} \leq \epsilon_{0}$ and $y(t) \leq \epsilon_{1}$, for $t \in\left[s_{n}, t_{n}\right]$, we have

$$
\dot{y}(t) \geq y(t)\left[\frac{(-1)^{k}+1}{2}\left(-\tilde{a}_{1}-\tilde{b}_{1} \epsilon_{0}\right)+\frac{(-1)^{k+1}+1}{2}\left(-\tilde{a}_{2}-\tilde{b}_{2} \epsilon_{0}\right)\right]
$$

for any $t \in\left[s_{n}, t_{n}\right]$, we have

$$
\begin{aligned}
y(t) & \geq y\left(s_{n}\right) \exp \left\{\int_{s_{n}}^{t}\left[\frac{(-1)^{k}+1}{2}\left(-\tilde{a}_{1}-\tilde{b}_{1} \epsilon_{0}\right)+\frac{(-1)^{k+1}+1}{2}\left(-\tilde{a}_{2}-\tilde{b}_{2} \epsilon_{0}\right)\right] d v\right\} \\
& \geq \epsilon_{1} \exp \left\{\tilde{T}_{1}\left[\frac{(-1)^{k}+1}{2}\left(-\tilde{a}_{1}-\tilde{b}_{1} \epsilon_{0}\right)+\frac{(-1)^{k+1}+1}{2}\left(-\tilde{a}_{2}-\tilde{b}_{2} \epsilon_{0}\right)\right]\right\} \\
& \triangleq m_{2}^{*} .
\end{aligned}
$$

If $t_{n}-s_{n}>\tilde{T}_{1}$, then, for any $t \in\left[s_{n}, t_{n}\right]$, if $t \leq s_{n}+\tilde{T}_{1}$, then according to the above discussion in the case of $t_{n}-s_{n} \leq \tilde{T}_{1}$, we also have inequality (38). Particularly, we obtain $y\left(s_{n}+\tilde{T}_{1}\right) \geq$ $m_{2}^{*}$. Therefore, if $y(t) \leq \epsilon_{1}$ for all $t \in\left[s_{n}, t_{n}\right]$, from system (4), we have

$$
\begin{cases}\dot{x} \geq x\left(r_{1} \ln \frac{k_{1}}{x}-\xi_{0}\right), & t \in(2 k \omega,(2 k+1) \omega], \\ x\left(t^{+}\right)=\alpha_{1} x(t), & t=(2 k+1) \omega, \\ \dot{x} \geq x\left(a_{2}-b_{2} x-\xi_{0}\right), & t \in((2 k+1) \omega,(2 k+2) \omega], \\ x\left(t^{+}\right)=\alpha_{2} x(t), & t=(2 k+2) \omega .\end{cases}
$$

From (34), we can obtain

$$
x(t)>u_{0}^{*}(t)-\epsilon_{0}
$$

for all $t \in\left[s_{n}+\tilde{T}_{1}, t_{n}\right]$. Therefore, from system (4), we furthermore have

$$
\begin{aligned}
\dot{y}(t) \geq & y(t)\left[\frac{(-1)^{k}+1}{2}\left(-\tilde{a}_{1}+\tilde{c}_{1} \Phi_{1}\left(u_{0}^{*}(t)-\epsilon_{0}\right)-\tilde{b}_{1} \epsilon_{0}\right)\right. \\
& \left.+\frac{(-1)^{k+1}+1}{2}\left(-\tilde{a}_{2}+\tilde{c}_{2} \Phi_{2}\left(u_{0}^{*}(t)-\epsilon_{0}\right)-\tilde{b}_{2} \epsilon_{0}\right)\right]
\end{aligned}
$$

for all $t \in\left[s_{n}, t_{n}\right]$. For any $t \in\left[s_{n}+\tilde{T}_{1}, t_{n}\right]$, we firstly choose an integer $p \geq 0$ such that $t \in\left[s_{n}+\tilde{T}_{1}+2 p \omega, s_{n}+\tilde{T}_{1}+(2 p+2) \omega\right)$. Integrating inequality (41) from $s_{n}+\tilde{T}_{1}$ to $t$, from (38), we can obtain

$$
\begin{aligned}
y(t) \geq & y\left(s_{n}+\tilde{T}_{1}\right) \exp \left\{\int _ { s _ { n } + \tilde { T } _ { 1 } } ^ { t } \left[\frac{(-1)^{p}+1}{2}\left(-\tilde{a}_{1}+\tilde{c}_{1} \Phi_{1}\left(u_{0}^{*}(v)-\epsilon_{0}\right)-\tilde{b}_{1} \epsilon_{0}\right)\right.\right. \\
& \left.\left.+\frac{(-1)^{p+1}+1}{2}\left(-\tilde{a}_{2}+\tilde{c}_{2} \Phi_{2}\left(u_{0}^{*}(v)-\epsilon_{0}\right)-\tilde{b}_{2} \epsilon_{0}\right)\right] d v\right\} \\
\geq & m_{2}^{*} \exp \left\{\int _ { s _ { n } + \tilde { T } _ { 1 } } ^ { s _ { n } + \tilde { T } _ { 1 } + 2 p \omega } \left[\frac{(-1)^{p}+1}{2}\left(-\tilde{a}_{1}+\tilde{c}_{1} \Phi_{1}\left(u_{0}^{*}(v)-\epsilon_{0}\right)-\tilde{b}_{1} \epsilon_{0}\right)\right.\right. \\
& \left.+\frac{(-1)^{p+1}+1}{2}\left(-\tilde{a}_{2}+\tilde{c}_{2} \Phi_{2}\left(u_{0}^{*}(v)-\epsilon_{0}\right)-\tilde{b}_{2} \epsilon_{0}\right)\right] d v
\end{aligned}
$$




$$
\begin{aligned}
& +\int_{s_{n}+\tilde{T}_{1}+2 p \omega}^{t}\left[\frac{(-1)^{p}+1}{2}\left(-\tilde{a}_{1}+\tilde{c}_{1} \Phi_{1}\left(u_{0}^{*}(v)-\epsilon_{0}\right)-\tilde{b}_{1} \epsilon_{0}\right)\right. \\
& \left.\left.+\frac{(-1)^{p+1}+1}{2}\left(-\tilde{a}_{2}+\tilde{c}_{2} \Phi_{2}\left(u_{0}^{*}(v)-\epsilon_{0}\right)-\tilde{b}_{2} \epsilon_{0}\right)\right] d v\right\} \\
\geq & m_{2}^{*} \exp \left\{\int _ { s _ { n } + \tilde { T } _ { 1 } + 2 p \omega } ^ { t } \left[\frac{(-1)^{p}+1}{2}\left(-\tilde{a}_{1}+\tilde{c}_{1} \Phi_{1}\left(u_{0}^{*}(v)-\epsilon_{0}\right)-\tilde{b}_{1} \epsilon_{0}\right)\right.\right. \\
& \left.\left.+\frac{(-1)^{p+1}+1}{2}\left(-\tilde{a}_{2}+\tilde{c}_{2} \Phi_{2}\left(u_{0}^{*}(v)-\epsilon_{0}\right)-\tilde{b}_{2} \epsilon_{0}\right)\right] d v\right\} \\
\geq & m_{2}^{*} \exp \left\{-2 \omega\left[\frac{(-1)^{p}+1}{2}\left(\tilde{a}_{1}+\tilde{b}_{1} \epsilon_{0}\right)+\frac{(-1)^{p+1}+1}{2}\left(\tilde{a}_{2}+\tilde{b}_{2} \epsilon_{0}\right)\right]\right\} \\
= & \epsilon_{1} \exp \left\{-\left(\tilde{T}_{1}+2 \omega\right)\left[\frac{(-1)^{p}+1}{2}\left(\tilde{a}_{1}+\tilde{b}_{1} \epsilon_{0}\right)+\frac{(-1)^{p+1}+1}{2}\left(\tilde{a}_{2}+\tilde{b}_{2} \epsilon_{0}\right)\right]\right\} .
\end{aligned}
$$

Let $m_{2}=\epsilon_{1} \exp \left\{-\left(\tilde{T}_{1}+2 \omega\right)\left[\frac{(-1)^{p}+1}{2}\left(\tilde{a}_{1}+\tilde{b}_{1} \epsilon_{0}\right)+\frac{(-1)^{p+1}+1}{2}\left(\tilde{a}_{2}+\tilde{b}_{2} \epsilon_{0}\right)\right]\right\}$. Then, from the above discussion, we obtain

$$
y(t) \geq m_{2}
$$

for all $t \in \bigcup_{n=1}^{\infty}\left[s_{n}, t_{n}\right]$.

Therefore, for case 3 , we finally have

$$
y(t) \geq m_{2}
$$

for all $t \geq \tilde{T}_{2}$. Lastly, we consider case 2 . From $y(t) \geq \epsilon_{1}$ for all $t \geq T^{*}$, we directly obtain

$$
y(t) \geq m_{2},
$$

for all $t \geq T^{*}$, where $T^{*} \geq \tilde{T}_{2}+\tilde{T}_{1}$. Thus, we finally obtain

$$
\lim _{t \rightarrow \infty} \inf y(t) \geq m_{2}
$$

for any positive solution $y(t)$ of system (4).

This shows that $y(t)$ is permanent. This completes the proof of Theorem 3.3.

From the existence of impulsive periodic solution of Theorem 1 in [23], i.e., if an impulsive periodic system is permanent, then it has at least a periodic solution. We have the following result.

Corollary 3.4 If all assumptions of Theorem 3.3 hold, then there exists at least a $2 \omega$ periodic solution $\left(x^{*}(t), y^{*}(t)\right)$ of model (4).

Furthermore, we have the following result regarding the global attractivity of predatorfree periodic solution $\left(u_{0}^{*}(t), 0\right)$ of model (4).

Theorem 3.5 If $\left(H_{1}\right)$ and $\left(H_{2}\right)$ hold, and

$$
\int_{0}^{\omega}\left[-\tilde{a}_{1}+\tilde{c}_{1} \Phi_{1}\left(u_{0}^{*}(t)\right)\right] d t+\int_{\omega}^{2 \omega}\left[-\tilde{a}_{2}+\tilde{c}_{2} \Phi_{2}\left(u_{0}^{*}(t)\right)\right] d t \leq 0,
$$


then the predator-free periodic solution $\left(u_{0}^{*}(t), 0\right)$ of model $(4)$ is globally attractive, i.e., for any positive solution $(x(t), y(t))$ of model $(4)$, we have

$$
\lim _{t \rightarrow \infty} x(t)=u_{0}^{*}(t), \quad \lim _{t \rightarrow \infty} y(t)=0,
$$

where $u_{0}^{*}(t)$ is the unique periodic solution of model (5).

Proof From (46), we find that there is a constant $\epsilon_{1}>0$, such that

$$
\begin{aligned}
& \int_{0}^{\omega}\left[-\tilde{a}_{1}+\tilde{c}_{1} \Phi_{1}\left(u_{0}^{*}(t)\right)-\tilde{b}_{1} \epsilon_{1}\right] d t+\int_{\omega}^{2 \omega}\left[-\tilde{a}_{2}+\tilde{c}_{2} \Phi_{2}\left(u_{0}^{*}(t)\right)-\tilde{b}_{2} \epsilon_{1}\right] d t \\
& \quad \leq-\left(\tilde{b}_{1}+\tilde{b}_{2}\right) \epsilon_{1} \omega<0 .
\end{aligned}
$$

Furthermore, from the continuity of $\Phi_{1}(s)$ and $\Phi_{2}(s)$, there exists a small enough constant $\epsilon_{0}$ with $\epsilon_{0}<\epsilon_{1}$ such that

$$
\delta:=\int_{0}^{\omega}\left[-\tilde{a}_{1}+\tilde{c}_{1} \Phi_{1}\left(u_{0}^{*}(t)+\epsilon_{0}\right)\right] d t+\int_{\omega}^{2 \omega}\left[-\tilde{a}_{2}+\tilde{c}_{2} \Phi_{2}\left(u_{0}^{*}(t)+\epsilon_{0}\right)\right] d t \leq 0 .
$$

From system (4), we have

$$
\begin{cases}\dot{x} \leq r_{1} x \ln \frac{k_{1}}{x}, & t \in(2 k \omega,(2 k+1) \omega], \\ x\left(t^{+}\right)=\alpha_{1} x(t), & t=(2 k+1) \omega, \\ \dot{x} \leq x\left(a_{2}-b_{2} x\right), & t \in((2 k+1) \omega,(2 k+2) \omega], \\ x\left(t^{+}\right)=\alpha_{2} x(t), & t=(2 k+2) \omega .\end{cases}
$$

From Lemma 2.2, we have

$$
x(t) \leq u(t)
$$

for all $t \geq 0$, where $u(t)$ is the solution of the following system with initial condition $u(0)=$ $x(0)$ :

$$
\begin{cases}\dot{u}=r_{1} u \ln \frac{k_{1}}{u}, & t \in(2 k \omega,(2 k+1) \omega] \\ u\left(t^{+}\right)=\alpha_{1} u(t), & t=(2 k+1) \omega, \\ \dot{u}=u\left(a_{2}-b_{2} u\right), & t \in((2 k+1) \omega,(2 k+2) \omega] \\ u\left(t^{+}\right)=\alpha_{2} u(t), & t=(2 k+2) \omega\end{cases}
$$

By Lemma 2.1, we have

$$
\lim _{t \rightarrow \infty} u(t)=u_{0}^{*}(t)
$$

Therefore, for any constant $\epsilon>0$ with $\epsilon<\epsilon_{0}$, there exists a constant $T_{3}>T_{2}$ such that, for all $t \geq T_{3}$,

$$
x(t)<u_{0}^{*}(t)+\epsilon .
$$


Furthermore, from $\left(H_{1}\right)$ and (53), we have, for all $t \geq T_{3}$,

$$
\begin{aligned}
\dot{y}(t) \leq & y(t)\left\{\frac{(-1)^{k}+1}{2}\left[-\tilde{a}_{1}+\tilde{c}_{1} \Phi_{1}\left(u_{0}^{*}(t)+\epsilon_{0}\right)-\tilde{b}_{1} y(t)\right]\right. \\
& \left.+\frac{(-1)^{k+1}+1}{2}\left[-\tilde{a}_{2}+\tilde{c}_{2} \Phi_{2}\left(u_{0}^{*}(t)+\epsilon_{0}\right)-\tilde{b}_{2} y(t)\right]\right\} .
\end{aligned}
$$

If $y(t) \geq \epsilon_{1}$ for all $t \geq T_{3}$, we have

$$
\begin{aligned}
\dot{y}(t) \leq & y(t)\left\{\frac{(-1)^{k}+1}{2}\left[-\tilde{a}_{1}+\tilde{c}_{1} \Phi_{1}\left(u_{0}^{*}(t)+\epsilon_{0}\right)-\tilde{b}_{1} \epsilon_{1}\right]\right. \\
& \left.+\frac{(-1)^{k+1}+1}{2}\left[-\tilde{a}_{2}+\tilde{c}_{2} \Phi_{2}\left(u_{0}^{*}(t)+\epsilon_{0}\right)-\tilde{b}_{2} \epsilon_{1}\right]\right\} .
\end{aligned}
$$

For any $t \geq T_{3}$, we choose an integer $n_{t} \geq 0$ such that $t \in\left(2 n_{t} \omega+T_{3}, 2\left(n_{t}+1\right) \omega+T_{3}\right]$, then integrating (55) from $T_{3}$ to $t$, we can obtain

$$
\begin{aligned}
y(t) \leq & y\left(T_{3}\right) \exp \left\{\int _ { T _ { 3 } } ^ { t } \left[\frac{(-1)^{k}+1}{2}\left(-\tilde{a}_{1}+\tilde{c}_{1} \Phi_{1}\left(u_{0}^{*}(v)+\epsilon_{0}\right)-\tilde{b}_{1} \epsilon_{1}\right)\right.\right. \\
& \left.\left.+\frac{(-1)^{k+1}+1}{2}\left(-\tilde{a}_{2}+\tilde{c}_{2} \Phi_{2}\left(u_{0}^{*}(v)+\epsilon_{0}\right)-\tilde{b}_{2} \epsilon_{1}\right)\right] d v\right\} \\
= & y\left(T_{3}\right) \exp \left\{\int _ { T _ { 3 } } ^ { T _ { 3 } + 2 n _ { t } \omega } \left[\frac{(-1)^{k}+1}{2}\left(-\tilde{a}_{1}+\tilde{c}_{1} \Phi_{1}\left(u_{0}^{*}(v)+\epsilon_{0}\right)-\tilde{b}_{1} \epsilon_{1}\right)\right.\right. \\
& \left.+\frac{(-1)^{k+1}+1}{2}\left(-\tilde{a}_{2}+\tilde{c}_{2} \Phi_{2}\left(u_{0}^{*}(v)+\epsilon_{0}\right)-\tilde{b}_{2} \epsilon_{1}\right)\right] d v \\
& +\int_{T_{3}+2 n_{t} \omega}^{t}\left[\frac{(-1)^{k}+1}{2}\left(-\tilde{a}_{1}+\tilde{c}_{1} \Phi_{1}\left(u_{0}^{*}(v)+\epsilon_{0}\right)-\tilde{b}_{1} \epsilon_{1}\right)\right. \\
& \left.\left.+\frac{(-1)^{k+1}+1}{2}\left(-\tilde{a}_{2}+\tilde{c}_{2} \Phi_{2}\left(u_{0}^{*}(v)+\epsilon_{0}\right)-\tilde{b}_{2} \epsilon_{1}\right)\right] d v\right\} \\
\leq & y\left(T_{3}\right) \exp \left\{n_{t} \delta+2 M_{1}^{*} \omega\right\}
\end{aligned}
$$

where $M_{1}^{*}=\max _{0 \leq t \leq 2 \omega}\left\{-\tilde{a}_{1}+\tilde{c}_{1} \Phi_{1}\left(u_{1}^{*}(t)+\epsilon_{0}\right)-\tilde{b}_{1} \epsilon_{1},-\tilde{a}_{2}+\tilde{c}_{2} \Phi_{2}\left(u_{1}^{*}(t)+\epsilon_{0}\right)-\tilde{b}_{2} \epsilon_{1}\right\}$.

Because $n_{t} \rightarrow \infty$ as $t \rightarrow \infty$, from (48) and (56), we easily obtain $\lim _{t \rightarrow \infty} y(t)=0$, which leads to a contradiction with $y(t) \geq \epsilon_{1}$. Therefore, there exists a $t_{1}>T_{3}$ such that $y\left(t_{1}\right)<\epsilon_{1}$. Because $y(t)$ is continuous for all $t \geq 0$, if further there exists a $t_{3}>t_{1}$ such that $y\left(t_{3}\right)>$ $\epsilon_{1} \exp \left\{2 \omega M_{1}^{*}\right\}$, then there exists a $t_{2} \in\left(t_{1}, t_{3}\right]$ such that $y\left(t_{2}\right)=\epsilon_{1}$, and $y(t) \geq \epsilon_{1}$ for all $t \in$ $\left[t_{2}, t_{3}\right]$. When $t \in\left[t_{2}, t_{3}\right]$, we have

$$
\begin{aligned}
\dot{y}(t) \leq & y(t)\left\{\frac{(-1)^{k}+1}{2}\left(-\tilde{a}_{1}+\tilde{c}_{1} \Phi_{1}\left(u_{1}^{*}(t)+\epsilon_{0}\right)-\tilde{b}_{1} \epsilon_{1}\right)\right. \\
& \left.+\frac{(-1)^{k+1}+1}{2}\left(-\tilde{a}_{2}+\tilde{c}_{2} \Phi_{2}\left(u_{1}^{*}(t)+\epsilon_{0}\right)-\tilde{b}_{2} \epsilon_{1}\right)\right\} .
\end{aligned}
$$


Choose an integer $n \geq 0$ such that $t_{3} \in\left[t_{2}+2 n \omega, t_{2}+2(n+1) \omega\right)$. Integrating (55) on $\left[t_{2}, t_{3}\right)$, we have

$$
\begin{aligned}
y\left(t_{3}\right) \leq & y\left(t_{2}\right) \exp \left\{\int _ { t _ { 2 } } ^ { t _ { 3 } } \left[\frac{(-1)^{k}+1}{2}\left(-\tilde{a}_{1}+\tilde{c}_{1} \Phi_{1}\left(u_{1}^{*}(t)+\epsilon_{0}\right)-\tilde{b}_{1} \epsilon_{1}\right)\right.\right. \\
& \left.\left.+\frac{(-1)^{k+1}+1}{2}\left(-\tilde{a}_{2}+\tilde{c}_{2} \Phi_{2}\left(u_{1}^{*}(t)+\epsilon_{0}\right)-\tilde{b}_{2} \epsilon_{1}\right)\right] d v\right\} \\
= & y\left(t_{2}\right) \exp \left\{\int _ { t _ { 2 } } ^ { t _ { 2 } + 2 n \omega } \left[\frac{(-1)^{k}+1}{2}\left(-\tilde{a}_{1}+\tilde{c}_{1} \Phi_{1}\left(u_{1}^{*}(t)+\epsilon_{0}\right)-\tilde{b}_{1} \epsilon_{1}\right)\right.\right. \\
& \left.+\frac{(-1)^{k+1}+1}{2}\left(-\tilde{a}_{2}+\tilde{c}_{2} \Phi_{2}\left(u_{1}^{*}(t)+\epsilon_{0}\right)-\tilde{b}_{2} \epsilon_{1}\right)\right] d v \\
& \left.+\int_{t_{2}+2 n \omega}^{t_{3}}\left(-\tilde{a}_{2}+\tilde{c}_{2} \Phi_{2}\left(u_{1}^{*}(t)+\epsilon_{0}\right)-\tilde{b}_{2} \epsilon_{1}\right) d v\right\} \\
\leq & \epsilon_{1} \exp \left\{2 \omega M_{1}^{*}\right\},
\end{aligned}
$$

which leads to a contradiction. Therefore, we have finally

$$
y(t) \leq \epsilon_{1} \exp \left\{2 \omega M_{1}^{*}\right\}
$$

for all $t \geq t_{1}$. Because $M_{1}^{*}$ is bounded with $\epsilon_{0}$ and $\epsilon_{1}$ is small enough, from (59), we finally obtain

$$
\lim _{t \rightarrow \infty} y(t)=0 .
$$

Now, we prove $\lim _{t \rightarrow \infty} x(t)=u_{0}^{*}(t)$. For any constant $\epsilon>0$, there exists a constant $\xi_{0}>0$ such that, for all $t \in[0, \infty)$,

$$
u_{\xi_{0}}^{*}(t)>u_{0}^{*}(t)-\frac{\epsilon}{2}
$$

where $u^{*}(t)$ is the unique positive periodic solution of system (25) with $\xi=\xi_{0}$. From (60), there exists a constant $T_{4}>T_{3}$ such that

$$
y(t)<\frac{\xi_{0}}{M_{2}^{*}}
$$

for all $t \geq T_{4}$, where $M_{2}^{*}=\max _{s \in[0, M]}\left\{\dot{\Phi}_{1}(s), \dot{\Phi}_{2}(s)\right\}$. For any $t \geq T_{4}$, from mean-value theorem and $\left(H_{1}\right)$, we see that there exist a $\xi_{1} \in(0, x(t))$ and a $\xi_{2} \in(0, x(t))$ such that

$$
\Phi_{1}(x(t))=\Phi_{1}(x(t))-\Phi_{1}(0)=\dot{\Phi}_{1}\left(\xi_{1}\right) x(t)
$$

and

$$
\Phi_{2}(x(t))=\Phi_{2}(x(t))-\Phi_{2}(0)=\dot{\Phi}_{2}\left(\xi_{2}\right) x(t) .
$$


Therefore, from (4), (62)-(64), we can obtain for any $t \geq T_{4}$

$$
\begin{cases}\dot{x}=r_{1} x\left(\ln \frac{k_{1}}{x}-\dot{\Phi}_{1}\left(\xi_{1}\right) y\right) \geq r_{1} x\left(\ln \frac{k_{1}}{x}-\xi_{0}\right), & t \in(2 k \omega,(2 k+1) \omega], \\ x\left(t^{+}\right)=\alpha_{1} x(t), & t=(2 k+1) \omega, \\ \dot{x}=x\left(a_{2}-b_{2} x-\dot{\Phi}_{2}\left(\xi_{2}\right) y\right) \geq x\left(a_{2}-b_{2} x-\xi_{0}\right), & t \in((2 k+1) \omega,(2 k+2) \omega], \\ x\left(t^{+}\right)=\alpha_{2} x(t), & t=(2 k+2) \omega .\end{cases}
$$

From Lemma 2.2, we have

$$
x(t) \geq u(t)
$$

for all $t \geq T_{4}$, where $u(t)$ is the solution of system (25) and initial condition $u\left(T_{4}^{+}\right)=x\left(T_{4}^{+}\right)$. Since $\lim _{t \rightarrow \infty} u(t)=u_{\xi_{0}}^{*}(t)$, for any constant $\epsilon>0$, there exists a constant $T_{5}>T_{4}$ such that

$$
u(t)>u_{\xi_{0}}^{*}(t)-\frac{\epsilon}{2}
$$

for all $t \geq T_{5}$. Therefore, from (61), (66), and (67), we obtain

$$
x(t) \geq u_{0}^{*}(t)-\epsilon
$$

for all $t \geq T_{5}$. From (53) and (68), we finally have

$$
\left|x(t)-u_{0}^{*}(t)\right|<\epsilon
$$

for all $t \geq T_{5}$.

This shows that $\lim _{t \rightarrow \infty} x(t)=u_{0}^{*}(t)$. This completes the proof of Theorem 3.5.

Remark 3.1 From Theorems 3.3 and 3.5, we can see that the whole dynamics of system (4) is decided by two parts, i.e., the season $1(t \in(2 k \omega,(2 k+1) \omega])$ and season 2 $(t \in((2 k+1) \omega,(2 k+2) \omega])$. From Theorems 3.3 and 3.5 , we can also see that the survivor of populations $x$ and $y$ is determined by the integral

$$
\delta=\int_{0}^{\omega}\left[-\tilde{a}_{1}+\tilde{c}_{1} \Phi_{1}\left(u_{0}^{*}(t)\right)\right] d t+\int_{\omega}^{2 \omega}\left[-\tilde{a}_{2}+\tilde{c}_{2} \Phi_{2}\left(u_{0}^{*}(t)\right)\right] d t
$$

I.e., if $\delta>0$, system (4) is permanent, if $\delta \leq 0$, then the predator $y$ will tend to extinction, while the prey $x$ will tend to the predator-free periodic solution $u_{0}^{*}(t)$. Therefore, our model means that the compositive effect of season 1 and 2, i.e., the logistic and Gompertz growth functions and predating functional response $\Phi_{i}(s)(i=1,2)$, including the impulsive effect $\alpha_{i}$, jointly influence the survivor of system (4). It is different from the previous results in which the model was always assumed in one season, or determined only by one process.

Remark 3.2 Let $\delta=\delta_{1}+\delta_{2}$, where

$$
\begin{aligned}
& \delta_{1}=\int_{0}^{\omega}\left[-\tilde{a}_{1}+\tilde{c}_{1} \Phi_{1}\left(u_{0}^{*}(t)\right)\right] d t, \\
& \delta_{2}=\int_{\omega}^{2 \omega}\left[-\tilde{a}_{2}+\tilde{c}_{2} \Phi_{2}\left(u_{0}^{*}(t)\right)\right] d t .
\end{aligned}
$$


$\delta_{1}>0$ (or $\delta_{2}>0$ ) means that the net growth of predator y is positive in season 1 (or season 2 ), i.e., the population of predator $y$ is increasing in season 1 (or season 2). $\delta_{1} \leq 0$ (or $\delta_{2} \leq 0$ ) means that the net growth of predator $y$ is negative in season 1 (or season 2), i.e., the population of predator $y$ is decreasing in season 1 (or season 2). According to Remark 3.1, we have the following four cases:

Case 1 , if $\delta_{1}>0, \delta_{2}>0$, then system (4) is permanent (see Fig. 1a, 1b);

Case 2, if $\delta_{1}<0$ (or $\left.\delta_{1}>0\right), \delta_{2}>0$ (or $\delta_{2}<0$ ) and $\delta=\delta_{1}+\delta_{2}>0$, then system (4) is permanent (see Fig. 3a, 3b);

Case 3, if $\delta_{1} \leq 0, \delta_{2} \leq 0$, then the predator $y$ will tend to extinction, while the prey $x$ will tend to the predator-free periodic solution $u_{0}^{*}(t)$ (see Fig. 2a, 2b);

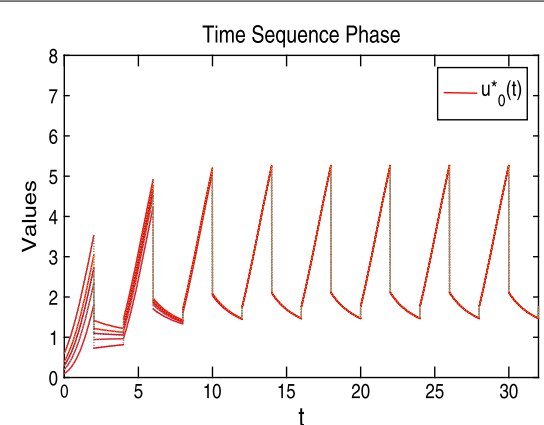

(1a)

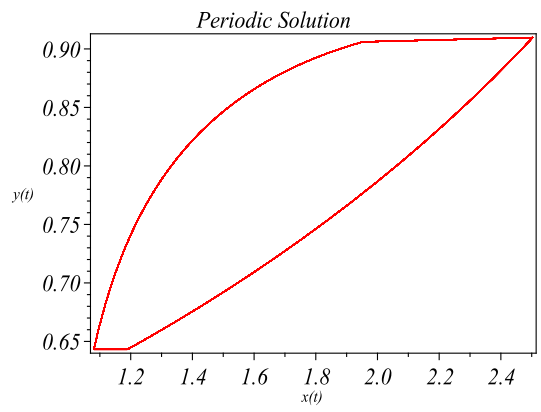

(1c)

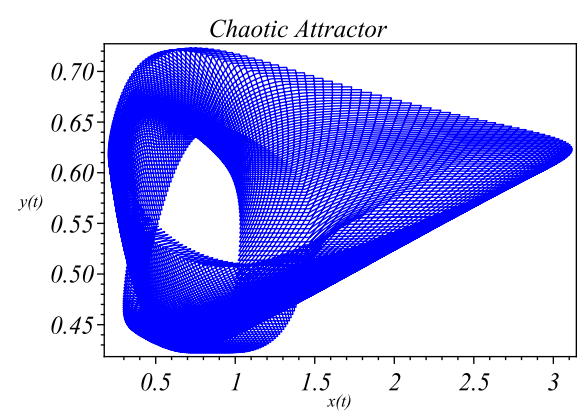

(1e)

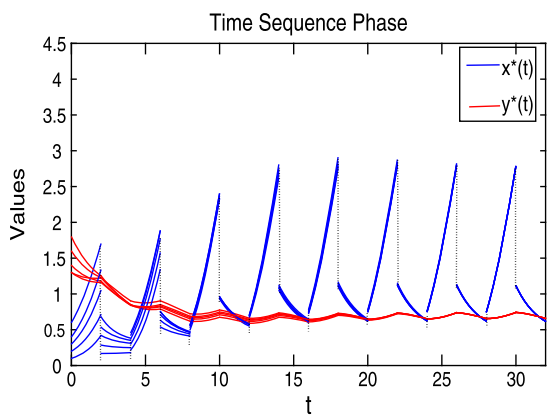

(1b)

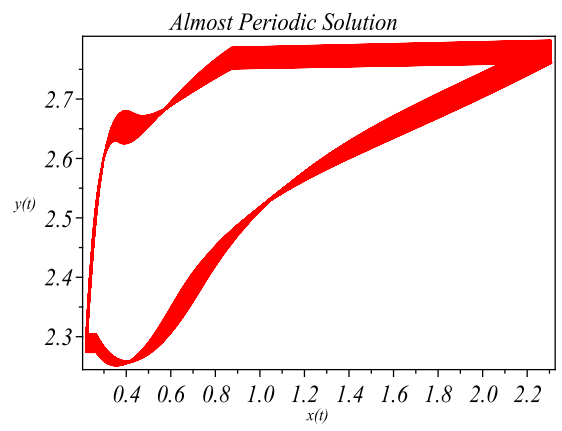

(1d)

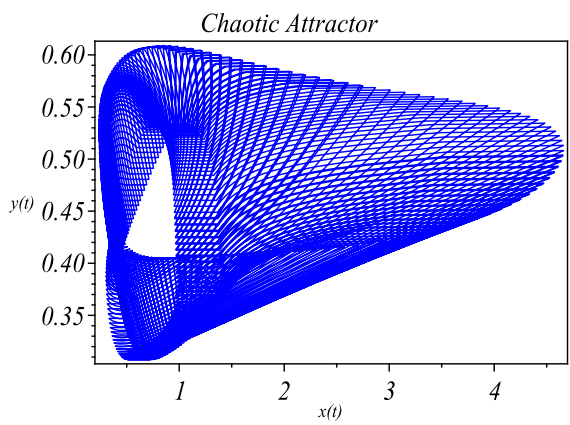

(1f)

Figure 1 Dynamics behavior of model (5) and (4); for model (5), we take initial values $x_{0}=0.1,0.2,0.3,0.4,0.6$, respectively; for model $(4)$, we take initial values $\left(x_{0}, y_{0}\right)=(0.1,1.8),(0.2,1.6),(0.3,1.4),(0.4,1.5),(0.6,1.3)$, respectively 


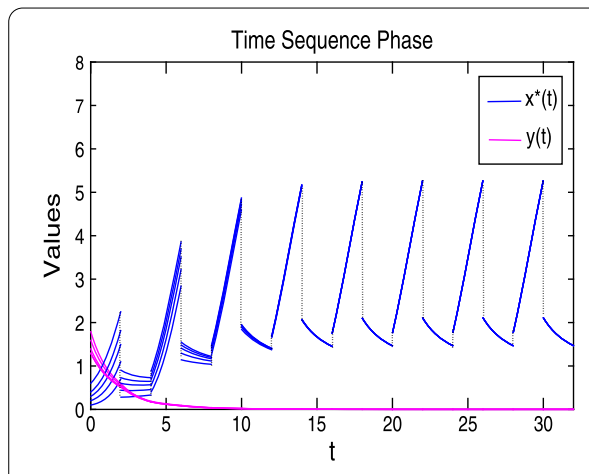

(2a)

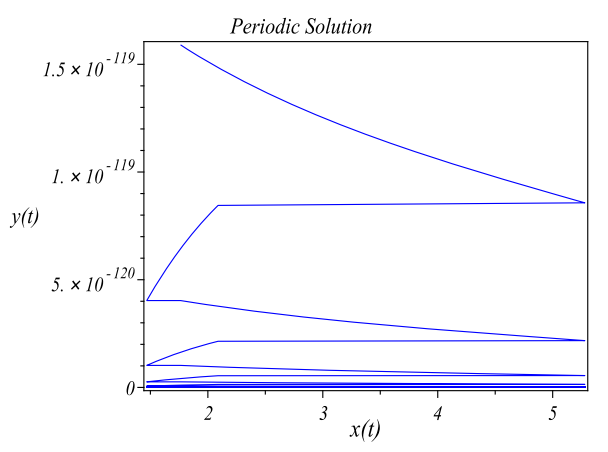

$(2 \mathrm{~b})$

Figure 2 Dynamics behavior of model (4); here we take initial values $\left(x_{0}, y_{0}\right)=(0.1,1.8),(0.2,1.6),(0.3,1.4)$, $(0.4,1.5),(0.6,1.3)$, respectively

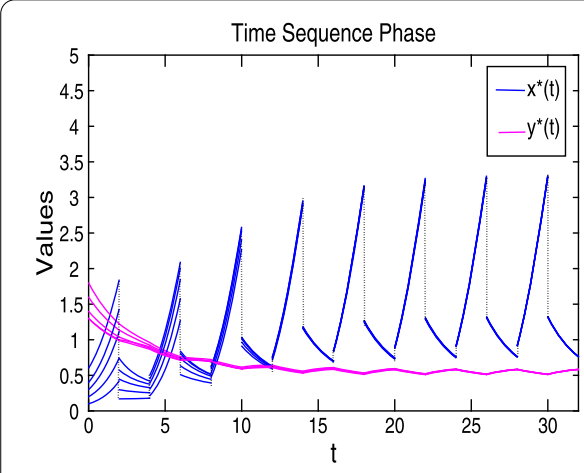

(3a)

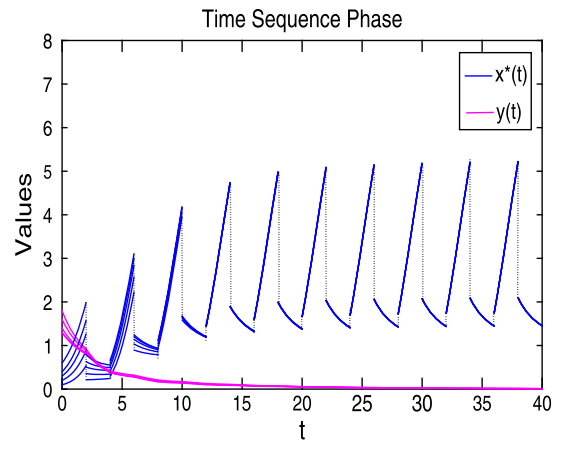

$(3 \mathrm{c})$

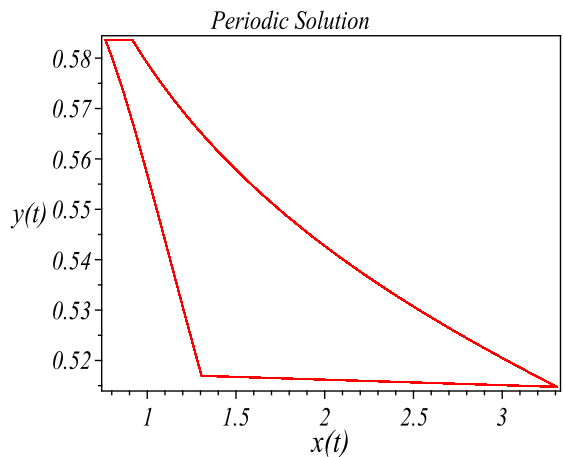

(3b)

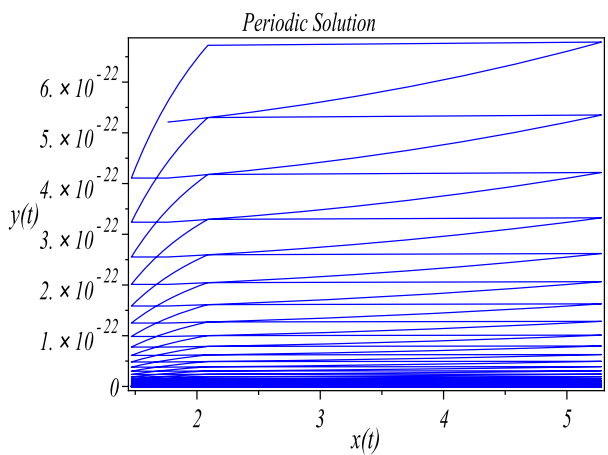

(3d)

Figure 3 Dynamics behavior of model (4); here we take initial values $\left(x_{0}, y_{0}\right)=(0.1,1.8),(0.2,1.6),(0.3,1.4)$ $(0.4,1.5),(0.6,1.3)$, respectively

Case 4, if $\delta_{1} \geq 0$ (or $\left.\delta_{1} \leq 0\right), \delta_{2} \leq 0$ (or $\delta_{2} \geq 0$ ) and $\delta=\delta_{1}+\delta_{2} \leq 0$, then the conclusion is as the same as the Case 3 (see Fig. 3c, 3d).

\section{Numerical simulation and discussion}

In this paper, we have investigated a class of two-species predator-prey models with two kinds of general functional responses and prey Gompertz and logistic growth alternating under seasonal succession. The criteria on the ultimate boundedness, permanence, and 
global attractivity of nonnegative periodic solution for model (4) are established. Biologically, we can see the effects of season succession on population dynamics due to changes of environment. E.g., there exists competition between two species in good season (or season with rich abundance of food), while without competition in the bad season (or season with severe scarcity of food; see, e.g., [7, 9]), meanwhile, the population in good season grows faster than in bad season (see, e.g., [10]).

To illustrate our results, in model (4), we take $\Phi_{1}(s)=\frac{c s}{e+s}, \Phi_{2}(s)=\frac{d s^{2}}{f+s^{2}}, r_{1}=0.5, a_{2}=0.25$, $b_{2}=0.25, k_{1}=10, c=1, e=1, d=1, f=1, \tilde{a}_{1}=0.1, \tilde{a}_{2}=0.1, \tilde{b}_{1}=0.1, \tilde{b}_{2}=0.1, \tilde{c}_{1}=0.38$, $\tilde{c}_{2}=0.28, \alpha_{1}=0.4$, and $\alpha_{2}=1.2$. In model (5), we take $r_{1}=0.5, a_{2}=0.25, b_{2}=0.25, k_{1}=10$, $\alpha_{1}=0.4, \alpha_{2}=1.2$, and $\omega=2$. For assumption $\left(H_{2}\right)$, we can obtain

$$
\begin{aligned}
\frac{\left(\frac{a_{2}}{b_{2}}\right)^{1-a}}{k_{1}^{1-a} b^{a}(b-1)^{1-a}} & \approx \frac{1}{10^{1-0.368} \times 1.649^{0.368} \times(1.649-1)^{1-0.368}} \approx 0.255 \\
& <\alpha_{1} \alpha_{2}^{a} \approx 0.4 \times 1.2^{0.368} \approx 0.428 \\
& \leq 1
\end{aligned}
$$

According to Lemma 2.1, system (5) has a unique $2 \omega$-periodic solution $u_{0}^{*}(t)$, which is globally asymptotically stable, see Fig. $1 \mathrm{a}$, in which we can see $1.4 \leq u_{0}^{*}(t) \leq 5.4$.

Furthermore, for condition (15), we can obtain

$$
\begin{aligned}
& \int_{0}^{\omega}\left[-\tilde{a}_{1}+\tilde{c}_{1} \Phi_{1}\left(u_{0}^{*}(t)\right)\right] d t+\int_{\omega}^{2 \omega}\left[-\tilde{a}_{2}+\tilde{c}_{2} \Phi_{2}\left(u_{0}^{*}(t)\right)\right] d t \\
& \quad \geq \int_{0}^{2}\left[-0.1+0.38\left(\frac{1.4}{1.4+1}\right)\right] d t+\int_{2}^{4}\left[-0.1+0.28\left(\frac{1.96}{1.96+1}\right)\right] d t \\
& \quad \approx 0.243+0.171 \approx 0.414>0 .
\end{aligned}
$$

Therefore, from Theorem 3.3 and Corollary 3.4, we can see that system (5) is permanent and has a periodic solution $\left(x^{*}(t), y^{*}(t)\right)$ (see Fig 1b, 1c).

In addition, if we take the nutrition conversion rate $\tilde{c}_{1}=0.08, \tilde{c}_{2}=0.28$, the death rate of predator species $\tilde{a}_{1}=0.06, \tilde{a}_{2}=0.02$, and the other parameters are constant as mentioned in Fig. 1, for condition (15), we can obtain

$$
\begin{aligned}
& \int_{0}^{\omega}\left[-\tilde{a}_{1}+\tilde{c}_{1} \Phi_{1}\left(u_{0}^{*}(t)\right)\right] d t+\int_{\omega}^{2 \omega}\left[-\tilde{a}_{2}+\tilde{c}_{2} \Phi_{2}\left(u_{0}^{*}(t)\right)\right] d t \\
& \quad \geq \int_{0}^{2}\left[-0.06+0.08\left(\frac{1.4}{1.4+1}\right)\right] d t+\int_{2}^{4}\left[-0.02+0.28\left(\frac{1.96}{1.96+1}\right)\right] d t \\
& \quad \approx-0.027+0.331 \approx 0.304>0 .
\end{aligned}
$$

Therefore, from Theorem 3.3 and Corollary 3.4, we can see that system (4) is also permanent and has a positive $2 \omega$-periodic solution $\left(x^{*}(t), y^{*}(t)\right)$, which is globally attractive (Fig. 3a, 3b). Here, although the results are as the same as above case, they are different. The condition (15) in the former is $\delta_{1}>0, \delta_{2}>0$, but in the latter, condition (15) is $\delta_{1}<0$, $\delta_{2}>0$ and $\delta=\delta_{1}+\delta_{2}>0$. That means the net growth rate $\delta$ of predator $y$ is both positive in seasons 1 and 2 in the former case, but is only positive in the season 2 and negative in the season 1 in the latter case. Therefore, our results mean that the survival of populations $x$ 
and $y$ is determined by the two parts of seasons 1 and 2 together, though the growth rate $\delta_{i}(i=1,2)$ of predator $y$ maybe be negative in any one season, but if the multiple effects of $\delta=\delta_{1}+\delta_{2}>0$, then both populations $x$ and $y$ will be permanent, which is different from the previous results (see, e.g., $[15,16]$ )

Furthermore, if we take $T=4.001$ and $T=4.02$, with other parameters unchanged, from numerical simulations (Fig. 1d, e, and f), we can see that population dynamics change from periodic to almost periodic to chaotic. We find that the population dynamics of system (4) is so sensitive with the actual parameters of ecosystems that any kinds of tiny changes in the environment can cause different types of population trajectories.

Moreover, if we take $\tilde{a}_{1}=0.5, \tilde{a}_{2}=0.6, \tilde{c}_{1}=0.25, \tilde{c}_{2}=0.3$, and the other parameters unchanged as above mentioned in Fig. 1, for condition (42), we can obtain

$$
\begin{aligned}
& \int_{0}^{\omega}\left[-\tilde{a}_{1}+\tilde{c}_{1} \Phi_{1}\left(u_{0}^{*}(t)\right)\right] d t+\int_{\omega}^{2 \omega}\left[-\tilde{a}_{2}+\tilde{c}_{2} \Phi_{2}\left(u_{0}^{*}(t)\right)\right] d t \\
& \quad \leq \int_{0}^{2}\left[-0.5+0.25\left(\frac{5.4}{5.4+1}\right)\right] d t+\int_{2}^{4}\left[-0.6+0.3\left(\frac{29.16}{29.16+1}\right)\right] d t \\
& \quad \approx(-0.578)+(-0.620) \approx-1.198<0 .
\end{aligned}
$$

Therefore, from Theorem 3.5, we can see that the predator $y$ of system (4) is extinct, while the prey $x$ will tend to the predator-free $2 \omega$-periodic solution $\left(u_{0}^{*}(t), 0\right)$ (see Fig $2 \mathrm{a}, 2 \mathrm{~b}$ ).

In a brief, the population dynamics of two-species predator-prey model with alternating Gompertz and logistic growth of prey under seasonal succession, and general functional responses of predation are more complicated and subtle with the changes of environment. Therefore, we conclude that the hybrid population models under seasonal succession permit more possibilities of survival of life, which is more consistent with the real ecosystem than the usual studied population models, and could be a new choice to model nature.

\section{Funding}

This work was supported by The National Natural Science Foundation of P.R. China (11861065, 11361059, 11271312 11702237), the Natural Science Foundation of Xinjiang (Grant No. 2019D01C076, 2017D01C082), the Scientific Research Program of the Higher Education Institution of Xinjiang (Grant No. XJEDU2017T001), The Scientific Research Project of Xinjiang university (BS160204).

\section{Competing interests}

The authors declare that there is no conflict of interest regarding the publication of this paper

\section{Authors' contributions}

LH mainly finished the writing of the whole content of the paper. LZ and ZT mainly finished the establishment of model and development. XW and HL mainly finished the numerical simulations. All authors read and approved the final manuscript.

\section{Publisher's Note}

Springer Nature remains neutral with regard to jurisdictional claims in published maps and institutional affiliations.

Received: 9 September 2019 Accepted: 19 December 2019 Published online: 03 January 2020

\section{References}

1. Moulin, T., Perasso, A., Gillet, F.: Modelling vegetation dynamics in managed grasslands: responses to drivers depend on species richness. Ecol. Model. 374, 22-36 (2018)

2. Fujiki, T., Sasaoka, K., Matsumoto, K., Wakita, M., et al.: Seasonal variability of phytoplankton community structure in the subtropical western North Pacific. J. Oceanogr. 72(3), 343-358 (2016)

3. Herb, W.R., Stefan, H.G.: Seasonal growth of submersed macrophytes in lakes: the effects of biomass density and light competition. Ecol. Model. 193(3-4), 560-574 (2006)

4. Levy, D., Harrington, H.A., Van Gorder, R.A.: Role of seasonality on predator-prey-subsidy population dynamics. J. Theor. Biol. 396, 163-181 (2016) 
5. Klaumeier, C.A.: Floquet theory: a useful tool for understanding nonequilibrium dynamics. Theor. Ecol. 1, 153-163 (2008)

6. Jennifer, J.H., Jonathan, A., Andrew, W., et al.: A comparison of the dynamical impact of seasonal mechanisms in a herbivore-plant defence system. Theor. Ecol. 6(2), 225-239 (2013)

7. Li, Y.Q., Zhang, L., Teng, Z.D.: Single-species model under seasonal succession alternating between Gompertz and logistic growth and impulsive perturbations. GEM Int. J. Geomath. 8(2), 241-260 (2017)

8. Steiner, C.E., Schwaderer, A.S., Huber, V., et al.: Periodically forced food chain dynamics: model predictions and experimental validation. Ecology 90(11), 3099-3107 (2009)

9. Li, J.X., Zhao, A.M.: Stability analysis of a non-autonomous Lotka-Volterra competition model with seasonal succession. Appl. Math. Model. 40(2), 763-781 (2016)

10. Hsu, S.B., Zhao, X.Q.: A Lotka-Volterra competition model with seasonal succession. J. Math. Biol. 64(1), 109-130 (2012)

11. Caicedo, A., Cruz, F.W., Limeira, R., et al.: A diffusive logistic equation with concentrated and nonlocal sources. Math. Methods Appl. Sci. 40(16), 5975-5985 (2017)

12. Korobenko, L., Braverman, E.: On logistic models with a carrying capacity dependent diffusion: stability of equilibria and coexistence with a regularly diffusing population. Nonlinear Anal., Real World Appl. 13(6), 2648-2658 (2012)

13. Zhou, P., Xiao, D.M.: The diffusive logistic model with a free boundary in heterogeneous environment. J. Differ. Equ. 256(6), 1927-1954 (2014)

14. Yu, Y.M., Wang, W.D., Lu, Z.Y.: Global stability of Gompertz model of three competing populations. J. Math. Anal. Appl. 334(1), 333-348 (2007)

15. Zhang, L., Teng, Z.D., Liu, Z.J.: Survival analysis for a periodic predator-prey model prey impulsively unilateral diffusion in two patches. Appl. Math. Model. 35(9), 4243-4256 (2011)

16. Zhang, L., Teng, Z.D.: The dynamical behavior of a predator-prey system with Gompertz growth function and impulsive dispersal of prey between two patches. Math. Methods Appl. Sci. 39, 3623-3639 (2016)

17. Tang, S., Chen, L.S.: Density-dependent birth rate, birth pulses and their population dynamic consequences. J. Math. Biol. 44(2), 185-199 (2002)

18. Pei, Y.Z., Zeng, G.Z., Chen, L.S.: Species extinction and permanence in a prey-predator model with two-type functional responses and impulsive biological control. Nonlinear Dyn. 52(1-2), 71-81 (2008)

19. Lakshmikantham, V., Bainov, D.D., Simeonov, P.S.: Theory of Impulsive Differential Equations. World Scientific, Singapore (1989)

20. Bainov, D., Simeonov, P.: Impulsive Differential Equations: Periodic Solutions and Applications. Longman, London (1993)

21. Hu, H.X., Xu, L.G.: Existence and uniqueness theorems for periodic Markov process and applications to stochastic functional differential equations. J. Math. Anal. Appl. 466(1), 896-926 (2018)

22. Xu, L.G., Hu, H.X.: Exponential ultimate boundedness of non-autonomous fractional differential systems with time delay and impulses. Appl. Math. Lett. 99, 106000 (2020)

23. Teng, Z.D., Nie, L.F., Fang, X.N.: The periodic solutions for general periodic impulsive population systems of functional differential equations and its applications. Comput. Math. Appl. 61(9), 2690-2703 (2011)

\section{Submit your manuscript to a SpringerOpen ${ }^{\circ}$ journal and benefit from:}

- Convenient online submission

- Rigorous peer review

- Open access: articles freely available online

- High visibility within the field

- Retaining the copyright to your article

Submit your next manuscript at $\gg$ springeropen.com 